\title{
Effect of Heterogeneity on Formation of Shear Zones in Granular Bodies
}

\author{
Jacek Tejchman \\ Civil Engineering Department, Gdansk University of Technology, \\ 80-952 Gdansk, Poland \\ tejchmk@pg.gda.pl
}

\begin{abstract}
Heterogeneity of granular materials triggers shear zone formation. In the paper, the FE-analysis of the effect of heterogeneity on the formation of a spontaneous shear zone inside of granular material during a plane strain compression test is presented. The numerical calculations are performed with a finite element method on the basis of a hypoplastic constitutive law extended by polar quantities: rotations, curvatures, couple stresses and a mean grain diameter used as a characteristic length. The heterogeneity in the granular body is assumed in the form of spots with a different initial void ratio. The spots are single and distributed randomly or stochastically.
\end{abstract}

\section{Introduction}

Localization of deformation in narrow shear zones is a fundamental phenomenon of granular material behaviour. Since shear localization is a precursor of failure of soils, it has to be taken into account when modelling granular materials. Therefore, the constitutive law has to include a characteristic length to describe the thickness of shear zones and a spacing between them. The experimental results show that the thickness of shear zones depends on many different factors as: pressure level, void ratio, direction of deformation, specimen size, mean grain diameter, grain roughness, grain hardness, surface roughness and stiffness of the surrounding structure in contact with a granular body. In addition, the distribution of microscopic inhomogeneities inherently present in granular materials and triggering shear localization seems to be an influential factor [1]-[3]. Thus, while modeling shear localization it is of major importance to use a constitutive model taking all influential factors into account.

The intention of the paper was to show the effect of the distribution of heterogeneity on the formation of shear localization during a plane strain compression test with dry cohesionless sand specimen. The FE-calculations were performed with a polar hypoplastic constitutive model [4]-[6]. In this model, a characteristic length is directly related to the mean grain diameter of the granulate. 


\section{Constitutive Law and FE-Input Data}

The polar hypoplastic constitutive model [4]-[6] can reproduce essential features of granular bodies during shear localization depending on the void ratio, pressure level, direction of the deformation and curvature rate, mean grain diameter and grain roughness. The material parameters can be found by means of standard element tests and simple index tests. They are correlated with grain properties, i.e. they can be estimated from granulometric properties encompassing grain size distribution curve, shape, angularity and hardness of grains. The FE-analyses were carried out with the following material constants (for so-called Karlsruhe sand): $e_{i 0}=1.3, e_{d 0}=0.51, e_{c 0}=0.82$, $\phi_{c}=30^{\circ}, h_{s}=190 \mathrm{MPa}, n=0.5, \alpha=0.3, d_{50}=0.5 \mathrm{~mm}, a_{c}=a_{1}^{-1}[4]$.

The FE-calculations of plane strain compression tests were performed with a sand specimen which was $h_{o}=10 \mathrm{~cm}$ high and $b=2 \mathrm{~cm}$ wide (length $l=1.0 \mathrm{~m}$ ).

A quasi-static deformation in sand was initiated through a constant vertical displacement increment prescribed at nodes along the upper edge of the specimen. The boundary conditions of the sand specimen were no shear stress and couple stress at the top and bottom. To preserve the stability of the specimen against the sliding along the bottom boundary, the node in the middle of the bottom was kept fixed.

To obtain a shear zone inside the granular specimen, single weaker and stronger spots and spots distributed randomly or stochastically were assumed. The single spots were inserted into the specimen in the form of a weaker finite element (with a higher initial void ratio) or a stronger finite element (with a lower initial void ratio). The location and the distance of imperfect elements varied.

The effect of a distribution of the initial void ratio $e_{o}$ in the entire specimen was also investigated. In the first case, the initial void ratio $e_{o}$ was randomly distributed (by means of a random generator) in such a way that the initial void ratio was increased in every element layer by the value $a \times r$, where $a$ is a constant (e.g. 0.0001, $0.05)$ and $r$ is a random number between 0 and 1 .

In addition, the initial void ratio was distributed stochastically with an exponential frequency function proposed by Shahinpoor [3], [7]:

\section{FE-Results}

The effect of the spacing of weaker elements in the dense specimen is demonstrated in Fig.1. Three weak elements $\left(e_{0}=0.90\right)$ are located uniformly along the left side of the specimen at distance of about $25 \mathrm{~mm}$. At the beginning, two shear zones always emerge expanding outward from each weaker element. Afterwards and up to the end, only one shear zone dominates (starting from the lowest weaker spot). It forms intensively between $u / h_{0}=2.25 \%$ and $u / h_{0}=2.84 \%$. The remaining shear zones become passive at the beginning of intense shearing in the dominated shear zone i.e. at $u / h_{0}=2.25 \%$. If the weaker elements are very close to each other, the shear zone forms at the same place. In contrast to FE-calculations of simple shearing of an infinite granular layer by Shi and Chang [8], the thickness of the shear zone does not grow and is the same (independently of the spacing of weak spots). 


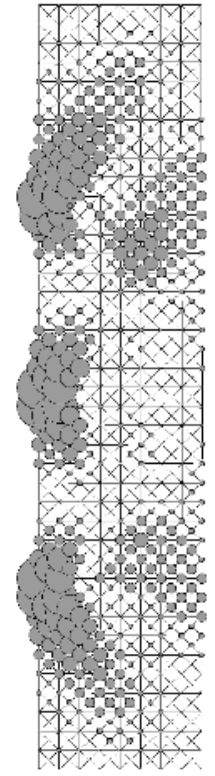

a)

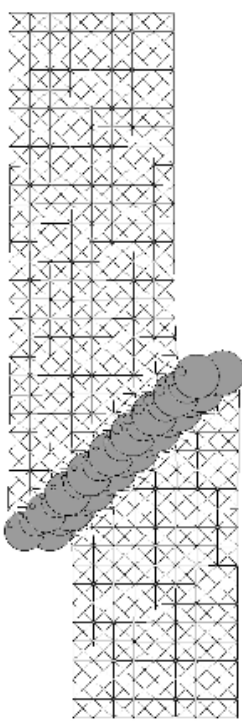

b)

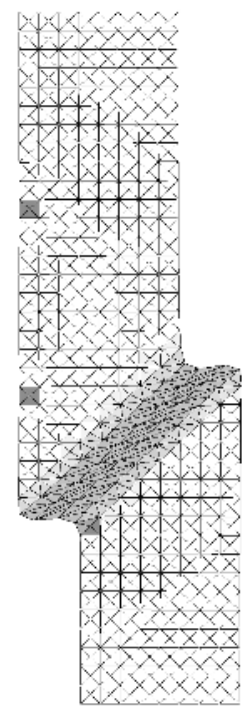

c)

Fig. 1. Evolution of modulus of the deformation rate $d$ in 3 weaker elements (a), deformed FEmesh with the distribution of Cosserat rotation $\omega^{f}$ at $u / h_{0}=2.5 \%$ (b) and Cosserat rotation $\omega$ and void ratio $e$ at $u / h_{0}=10 \%$ (c)

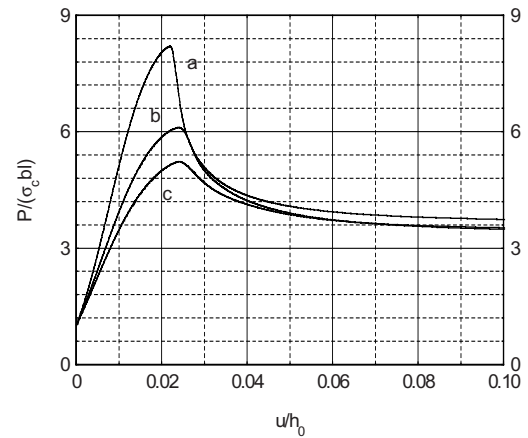

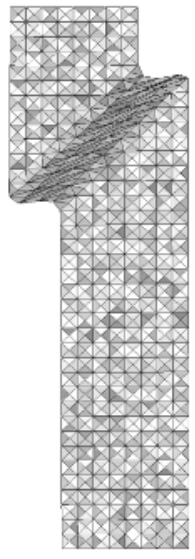

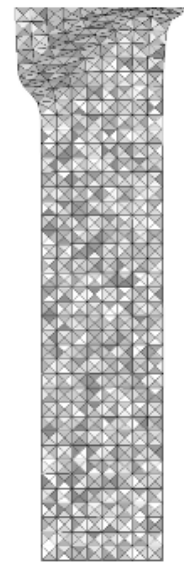

c)

Fig. 2. FE-results: load-displacement curves and deformed FE-mesh with a distribution of void ratio at residual state (exponential frequency distribution of the initial void ratio):

a) $\left.\bar{e}_{0}=0.55, \mathrm{~b}\right) \bar{e}_{0}=0.60$ an $\left.\mathrm{d} \mathrm{c}\right) \bar{e}_{0}=0.65$ ( $\bar{e}_{0}-$ mean void ratio $)$ 
The FE-results with a stochastic distribution of the initial void ratio in dense specimen (with a different mean void ratio) are shown in Fig.2.

As in the case of a random distribution of the initial void ratio, the shear zone can propagate in different parts of the granular specimen. The thickness of the shear zone slightly increases with increasing mean void ratio. Thus, this increase is significantly smaller as compared to the calculations with a random distribution of the initial void ratio (since the rate of softening is different in both cases).

\section{Conclusions}

Each single imperfection (weaker and stronger spot) induces shear zones at the beginning of loading independently of the initial density of the specimen. Thus, multiple shear zones appear which are not well developed.

The dominant single shear zone starts to form intensively in the dense specimen slightly before the peak state. It is well developed after the peak state.

The dominant shear zone is connected to a weaker spot but not to a stronger one.

The spacing of single imperfections does not influence the thickness of the shear zone. However, it influences the direction of propagation.

The distribution of the initial void ratio strongly influences the location of the shear zone and its propagation direction. The shear zone can appear in different parts of the dense specimen.

The results with a random distribution of the initial void ratio are similar as those with a stochastic distribution using an exponential frequency function.

\section{References}

1. Hobbs, B. E., Ord, A.: Numerical simulation of shear band formation in frictional-dilational materials. Ingenieur-Archiv 59 (1989) 209-220

2. Tejchman, J., Wu, W.: Numerical study on shear band patterning in a Cosserat continuum. Acta Mechanica 99 (1993) 61-74

3. Nübel, K., Karcher, C.: FE simulations of granular material with a given frequency of distribution of voids as initial condition. Granular Matter 1, 3 (1998) 105-112

4. Tejchman, J., Herle, I., Wehr, J.: FE-studies on the influence of initial void ratio, pressure level and mean grain diameter on shear localisation. Int. J. Num. Anal. Meth. Geomech. 23 (1999) 2045-2074

5. Tejchman, J., Gudehus, G.: Shearing of a narrow granular strip with polar quantities. Int. J. Num. and Anal. Methods in Geomechanics 25 (2001) 1-18.

6. Tejchman, J.: Patterns of shear zones in granular materials within a polar hypoplastic continuum. Acta Mechanica 155, 1-2 (2002) 71-95

7 Shahinpoor, M.: Statistical mechanical considerations on storing bulk solids. Bulk Solid Handling 1, 1 (1981) 31-36

8 Shi, Q., Chang, C. S.: Numerical analysis for the effect of heterogeneity on shear band formation. Proc. $16^{\text {th }}$ ASCE Eng. Mech. Conf., Seattle USA (2003) 1-11 